\title{
Array modeling and testing of fixed OWC type Wave Energy Converters
}

\author{
Bret Bosma, Ted Brekken, Pedro Lomonaco, Bryony DuPont, Chris Sharp, and Belinda Batten
}

\begin{abstract}
If wave energy technology is to mature to commercial success, array optimization could play a key role in that process. This paper outlines physical and numerical modeling of an array of five oscillating water column wave energy converters. Numerical model simulations are compared with experimental tank test data for a non-optimal and optimal array layout. Results show a max increase of $12 \%$ in average power for regular waves, and $7 \%$ for irregular waves between the non-optimized and optimized layouts. The numerical model matches well under many conditions; however, improvement is needed to adjust for phase errors. This paper outlines the process of numerical and physical array testing, providing methodology and results helpful for researchers and developers working with wave energy converter arrays.

Keywords-Oscillating water column, Wave energy converter array, Numerical modeling, Physical modeling
\end{abstract}

Manuscript received 16 March; accepted 27 March; published 30 November, 2020. This is an open access article distributed under the terms of the Creative Commons Attribution 4.0 licence (CC BY https://creativecommons.org/licenses/by/4.0/). This article has been subject to single-blind peer review by a minimum of two reviewers. This paper is based upon work supported by the US DoE under Award Number DE-EE0006816. Neither the US Government nor any agency thereof, nor any of their employees, makes any warranty, expressed or implied, or assumes any legal liability or responsibility for the accuracy, completeness, or usefulness of any information, apparatus, product, or process disclosed, or represents that its use would not infringe upon privately owned rights. Reference herein to any specific commercial product, process, or service by trade name, trademark, manufacturer, or otherwise does not necessarily constitute or imply its endorsement, recommendation, or favouring by the US Government or any agency thereof. The views and opinions of the authors expressed herein do not necessarily state or reflect those of the US Government or any agency thereof.

B. Bosma is with the O. H. Hinsdale Wave Research Laboratory, Oregon State University Corvallis, OR 97331 U.S.A (e-mail: bret.bosma@oregonstate.edu). T. Brekken is with the school of Electrical Engineering and Computer Science, Oregon State University 3025 Corvallis, OR 97331 U.S.A (e-mail: brekken@eecs.oregonstate.edu). P. Lomonaco is with the O. H. Hinsdale Wave Research Laboratory, Oregon State University Corvallis, OR 97331 U.S.A (e-mail: pedro.lomonaco@oregonstate.edu). B. DuPont is with the school of Mechanical, Industrial, and Manufacturing Engineering, Oregon State University Corvallis, OR 97331 U.S.A (e-mail: Bryony.DuPont@oregonstate.edu). C.Sharp was at the Pacific Marine Energy Center (PMEC), Oregon State University, Corvallis, OR, USA. He is now at the College of Engineering, George Fox University, Newberg, OR, USA (email: csharp@georgefox.edu). B. Batten is with the College of Engineering, Oregon State University Corvallis, OR 97331 U.S.A (e-mail: belinda.batten@oregonstate.edu). Digital Object Identifier https://doi.org/10.36688/imej.3.137-143

\section{INTRODUCTION}

$\mathrm{M}$ ANY contemporary visions of commercial wave energy production include array of devices working in concert. Current research in development of these arrays include device spacial placement and advance control techniques. Although related research was conducted for this project in these areas, this paper describes the software modeling and physical testing process of an array of Oscillating Water Columns (OWC) Wave Energy Converter (WEC) devices.

Much research into WEC arrays has occurred since the late 1970s with varied focus and conclusions. An overview of numerical modeling techniques is given in [1]. Other small array numerical studies are presented for OWC [2], and for generic devices in [3], [4]. Large array numerical modeling has been done for 9-25 devices in [5]-[7] and for over 1000 devices in [8]. Physical experimental array modeling has been done on OWC in [9] and heaving buoys in [10].

In this paper, modeling techniques are outlined and compared to preliminary results from the test data. The information presented here is part of a larger project on Advanced Laboratory and Field Arrays (ALFA), funded by the U.S. Department of Energy. Content will build upon a paper presented at EWTEC in 2017 [11] where a single device was modeled, tested, and characterized. Array placement decisions were based on research in genetic algorithms where initial results were also presented at EWTEC in 2017 [12].

\section{ALFA OVERVIEW}

The Pacific Marine Energy Center (PMEC) at Oregon State University is conducting research in WEC Array modeling, control, and placement. Under U.S. Department of Energy funding, an Advanced Laboratory and Field Arrays (ALFA) for Marine Energy project has been underway with several tasks. One task is performance enhancement for marine energy converters with several sub-tasks. Subtasks include WEC Array Design and Operations - Layout Optimization and Coordinated WEC Array Control. This paper describes the simulation, and physical model tank testing, of an array of fixed OWCs as part of the ALFA project. 


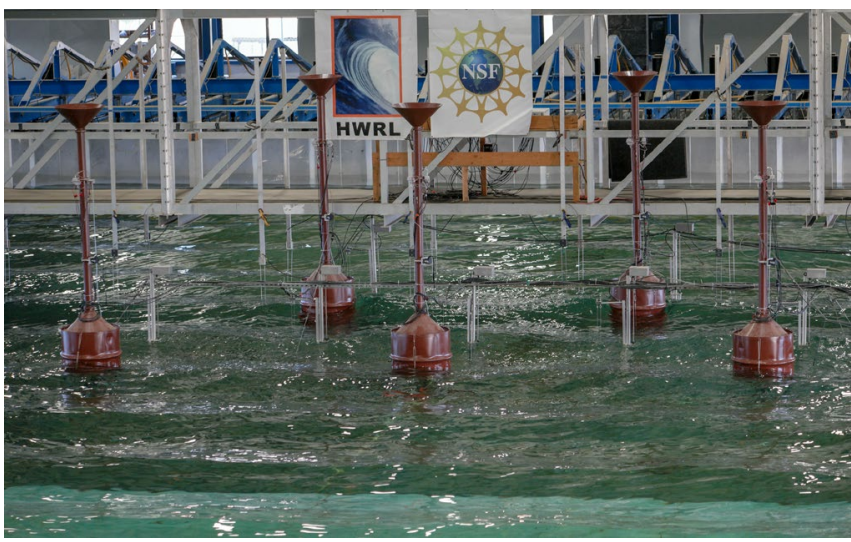

Fig. 1. Non-optimal array layout. Selected from literature search as a common array configuration.

Fixed OWCs were chosen as the test bed for the ALFA project because of their relatively simple geometry, low cost of fabrication, and ease of creating a computer simulation of the devices. The main structures of the physical devices are inexpensive recycled steel barrels. The air stack consists of easily fabricated automobile exhaust parts including pipes and flanges allowing for quick assembly and tight seals. Numerical modeling treated each OWC as a single heaving cylindrical point absorber. Initially, it was thought that there would be significant interaction between OWCs, however with the OWC spacing chosen, this paper will show that the interaction was small.

The details and characterization of the OWC physical parameters and operation is described in [11]. Each device was replicated and outfitted with identical hardware. This provides consistent results between devices.

A literature search was conducted, and popular array layout configurations were investigated. One layout, chosen from this study is shown in Fig. 1, which has the shape of a "W" with three OWC aligned in $x$ and separated by $3.6 \mathrm{~m}$ in $\mathrm{y}$, where $\mathrm{x}$ is in the direction of wave propagation and $\mathrm{y}$ is perpendicular in the horizonal plane. The remaining two OWC are then offset in $x$ by $3.7 \mathrm{~m}$ offshore between the three y locations. This was chosen as the non-optimal array configuration. Research into optimal spacing, when given a minimum separation distance, gave a layout of equal spacing in $\mathrm{y}$ and a

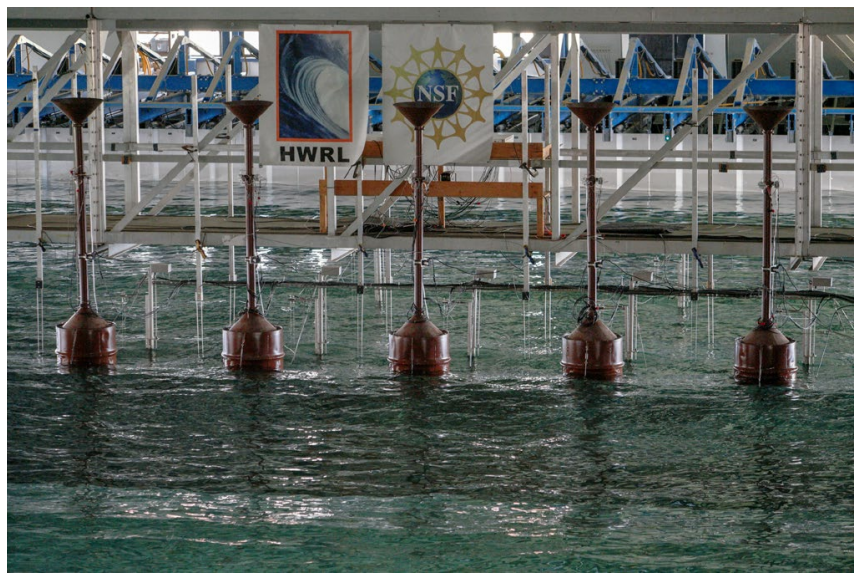

Fig. 2. Optimal array layout. Selected from optimization study.

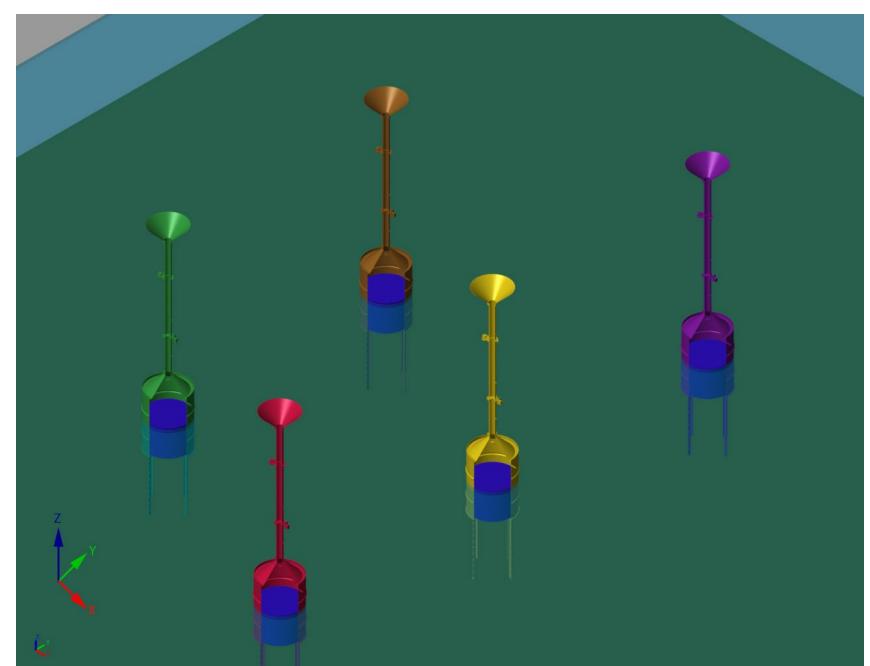

Fig. 3. WEC-Sim Non-optimal array layout. Blue cylinders are hydrodynamic bodies. Multi-colored bodies are for visualization only.

constant $x$ as shown in Fig. 2. In this paper, optimality is judged based on maximizing WEC power production.

\section{WEC-SIM MODEL}

A WEC-Sim [13] model of two array configurations was completed to assess the efficacy of predicting the physical tank test results. The non-optimal array spacing case WEC-Sim screen shot is shown in Fig. 3. The development path of the model followed a typical WECSim workflow with mesh creation, boundary element method software, and WEC-Sim steps. First, the geometry was created using a commercial 3D computer graphics program called Rhino [14]. Using Rhino allowed for a quality mesh to be generated which is key for accurate simulation. Each OWC body was saved as a .stl file and a .gdf file for WEC-Sim and WAMIT respectively. Next, WAMIT was run for the array under two physical layout configurations as described in section IV. BEMIO [15] was then used to evaluate the BEM results as well as prepare the data for the WEC-Sim simulation.

Generally, WEC-Sim solves the equation of motion of the WEC in six degrees of freedom

$$
m \ddot{X}=F_{\text {exc }}(t)+F_{\text {rad }}(t)+F_{P T O}(t)+F_{B}(t)
$$

where $F_{\text {exc }}$ is the excitation force, $F_{\text {rad }}$ is the radiation force, $F_{\text {PTO }}$ is the power take off force, and $F_{B}$ is the hydrostatic or buoyancy force. In order to include the fluid memory effect the Cummins formulation [16] for the radiation force is used.

$$
F_{r a d}(t)=-A_{\infty} \ddot{X}-\int_{0}^{t} K_{r}(t-\tau) \dot{X}(\tau) d \tau
$$

where $A_{\infty}$ is the added mass matrix at infinite frequency and $K_{r}$ is the radiation impulse response function.

Viscous, Morrison, and mooring forces were not considered for this study. The OWC modeled for this study is of a cylinder restricted to heave motion. 
Array interaction effects are computed in WAMIT. A good visualization of the interaction effects is found in the impulse response functions, namely the radiation force and excitation force. An important step in the numerical modeling process is to analyze the impulse response functions to determine whether the interaction effects are being captured as expected.

Fig. 4 shows the z-component radiation impulse response for each OWCs contribution to OWC A. Notice that OWC A radiation impulse response influence on OWC A occurs at time zero as expected and decays within a few seconds to zero. For the rest, the amplitude and time of occurance are both proportional to the distance from OWC A as expected. For example, OWC B contribution to OWC A has a greater amplitude and occurs in less time for the optimal layout case as compared to the non-optimal layout case.

Fig. 5 shows the z-component excitation impulse response function for each OWC. The excitation force impulse response is a non-causal system, meaning that the force influence from the incoming wave impacts the output before time zero. This is partially explained by the fact that WAMIT calculates the frequency domain data at the origin or some other specific point, however the incoming wave may impact the device prior to reaching this point [17]. Notice that for the non-optimal layout condition, where there is an offset in $\mathrm{x}$ for OWC B and D, the peak is shifted in time for those bodies. This is to be expected, however the oscillation before the peak is not expected and is likely an artifact from the WAMIT simulation. It may be possible to improve this response utilizing advanced techniques in WAMIT, however was not pursued for this project.

Input to the WEC-Sim model is a wave surface elevation time series. In all cases, the time series measured in the calibration phase of testing was used. For the calibration phase of testing, wave gauges were
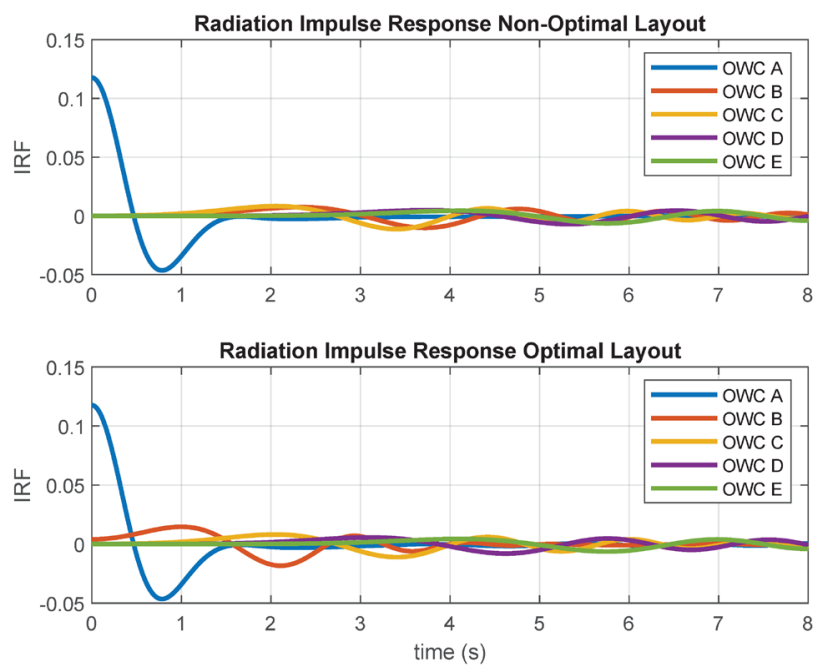

Fig. 4. Radiation Impulse Response functions on WEC A for non-optimal and optimal layouts. Notice that the amplitude and time of peak are dependent on the distance from WEC A as expected.

placed in the future locations of OWC and all wave conditions were run. This provided an opportunity to
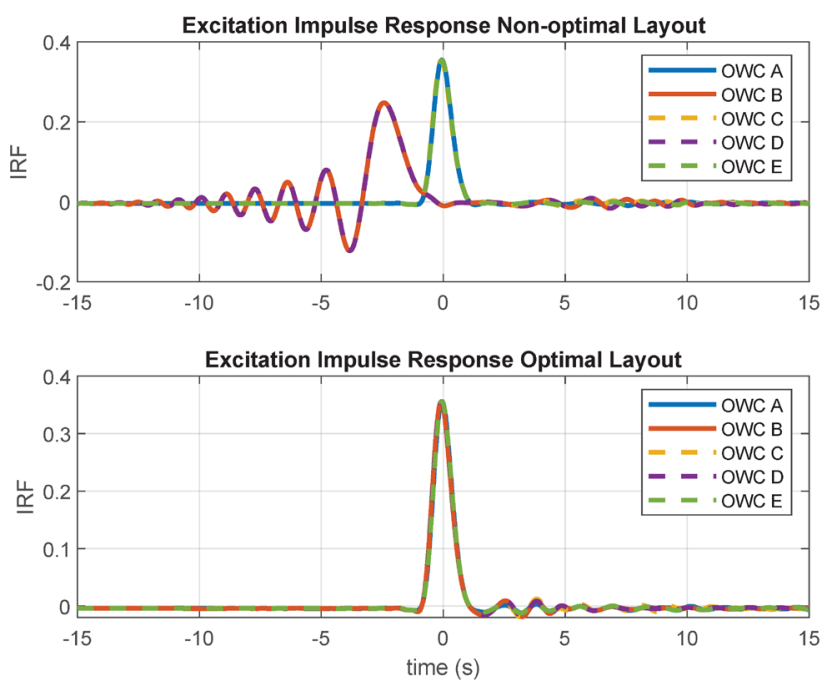

Fig. 5. Heave excitation impulse response for each body in the non-optimal and optimal array configuration.

compare the time series between simulation and experimental results.

\section{ARRAY TANK TESTING}

An array of OWC devices were designed built and tested as part of the ALFA project at Oregon State University. All wave tank tests were performed at the O.H. Hinsdale Wave research laboratory.

\section{A. Test facility}

The wave basin is $48.8 \mathrm{~m}$ long and $26.5 \mathrm{~m}$ wide and the water depth for all tests was $1.36 \mathrm{~m}$. The basin has 29 individual vertically hinged paddles and can create multidirectional waves. Fig. 1 and Fig. 2 show the device under test in the laboratory. Fig. 6 shows the locations of wave gauges and OWC for the tests performed. The origin is defined at the zero position of the wave board in $\mathrm{x}$. The basin has an instrument bridge off which a series of wave gauges were installed. It required three positions in order to cover the area shown. This not only allowed for the coverage area shown, but also provided repetition of tests for the PTO and other wave gauges, in order to verify the repeatability of the measurements. For calibration of the waves, the selfcalibrating wave gauges were in the future positions of the OWC and then moved offshore, as shown, for the duration of the tests.

The green circles represent the locations of the OWC for optimal layout conditions. For the non-optimal layout conditions, OWC B and D were moved offshore as shown with the red circles. 


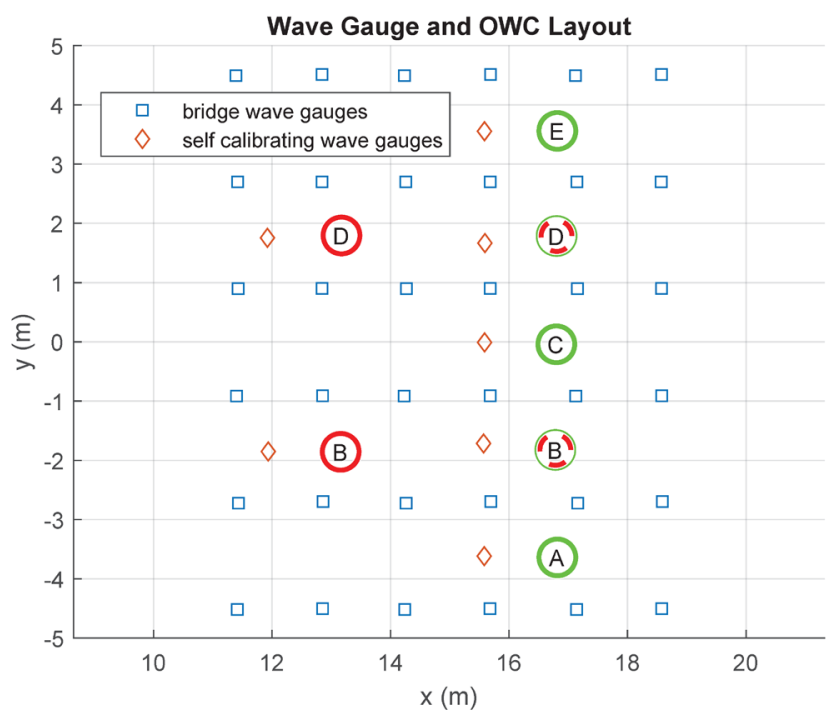

Fig. 6. Wave gauge OWC locations for tests. Bridge wave gauges are shown in blue squares corresponding to three bridge positions. Self-calibrating wave gauges were fixed for all tests. Green circles represent OWC locations for optimal layout. Red circles represent movement of OWC B and D for non-optimal layout.

\section{B. Control system and data acquisition}

The Power Take Off (PTO) of the OWC consists of a butterfly valve and orifice plate, which dissipate energy generated by the oscillating water column and measure air flow. Control of the butterfly valve is done with a stepper motor, which has a range of closed, minimal air flow, to open, maximum air flow. Each OWC has its own individual control system. Although the system is set up and capable of wave to wave scale control, for the tests reported here, the valve angle was set prior to the test and held for the duration of the test. Air flow was measured with the orifice plate for each device. Pressure sensors on each side of the plate allow for bidirectional flow measurements. Pressure drop between the main chamber and the ambient was used as the dynamic part of the PTO. Power was then computed as

$$
P_{\text {pto }}=p_{\text {chamber }} q_{v, o p}
$$

where $p_{\text {chamber }}$ is the pressure drop across the total PTO unit, and $q_{v, o p}$ is the volumetric flow of air measured through the orifice plate, which is assumed to be the same through the length of the system for each time step. More details of device construction and PTO system are found in [11]. All data was collected at a sampling rate of $100 \mathrm{~Hz}$.

\section{Test conditions}

Four combination of test conditions were identified of main interest for this study. An optimal and non-optimal layout, and optimal and non-optimal damping. For the non-optimal layout, a common array layout from a literature search was chosen. For the optimal layout, a genetic algorithm was used to select a layout under certain constraints as described in [12]. Constraints included the physical space in the basin, ranges of damping values that could be actuated in the devices, and minimum separation distances from a practical standpoint. For the non-optimal damping case, damping was optimized for a single device for the given wave condition, then applied equally to all five devices. For the optimal damping cases, damping was optimized for each individual WEC.

When this analysis was done, the solver identified unique damping values for each WEC. However, when these damping values were translated to valve angles there was very little difference between the non-optimal and optimal damping values. Instead of repeating the same tests again, the opportunity to try unique combinations of damping was used. Results from these unique combinations are omitted in this paper.

The wave conditions tested are shown in Table I. There are six regular wave conditions all with a wave height of $0.136 \mathrm{~m}$ and periods ranging from $1.22 \mathrm{~s}$ to 3.31 $\mathrm{s}$. Irregular waves included three with significant wave height of $0.136 \mathrm{~m}$ and periods ranging from $1.91 \mathrm{~s}$ to 2.61 $\mathrm{s}$, as well as a case with $0.242 \mathrm{~m}$ and $3.31 \mathrm{~s}$. These cases were uni-directional. The final case had significant wave height of $0.136 \mathrm{~m}$ and peak period of $1.91 \mathrm{~s}$ but was multidirectional with a spreading angle of 30 degrees.

For regular waves, the duration included time for the wave to propagate to the beach, back to the paddle, and back to the device location. At that point 20 wave cycles were run before a ramp down. All analysis was done on the 20 wave cycles after the initial transients. Irregular waves had a similar initial ramp up time, and the analysed test portion consisted of 600 waves for all wave cases. The spectral shape for all irregular wave cases followed a Pierson-Moscowitz spectral distribution.

\section{RESULTS}

The primary results shared in this paper are a comparison of WEC absorbed power for the various wave conditions and configurations. Details of the methods of analysis for a single OWC are provided in [11]. Before each wave run, the damping on all five OWC was set by fixing a known valve angle and holding it constant. For the WEC-Sim simulations, the calibrated wave surface elevation time series was input to the model. Regular wave input

A time series comparing the power results from a nonoptimized layout case for regular wave $H=0.136 \mathrm{~m}, T=$ $2.61 \mathrm{~s}$ as measured in the experiments, and the corresponding WEC-Sim case is shown in Fig. 7. Note that the WEC-Sim simulation OWC A, OWC C, and OWC $E$ amplitudes track reasonably well, however phase lags for OWC B and OWC D. For brevity, other time series have been omitted, however, other period waves showed a greater phase shift, suggesting that phase information is not properly accounted for in WAMIT as discussed in section III. Therefore, caution should be used if attempting to use WAMIT/WEC-Sim for array modeling where time series phase information is critical.

Focusing on average power values, Fig. 8 shows a comparison of average power with non-optimal layout on 
LayNoDamNo
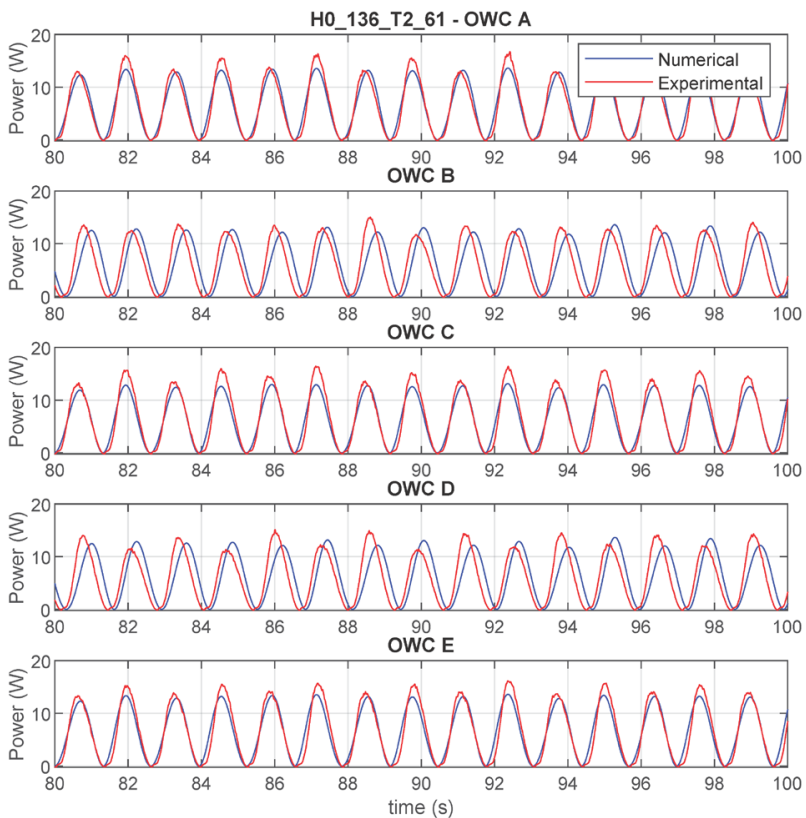

Fig. 7. Comparison of Power time series between experimental and numerical (WEC-Sim) results for a regular wave with $H=$ $0.136 \mathrm{~m}, T=2.61 \mathrm{~s}$. Amplitudes track reasonably well, however phase lags for OWC B and D, as an offset in $\mathrm{x}$.

the top row and optimal layout on the bottom row for each OWC. Bar graphs show the average of three bridge positions average power. Error bars show the minimum and maximum average power of the three bridge positions. Average power results show that the OWC operational range for power production have wave periods of $1.91 \mathrm{~s}, 2.26 \mathrm{~s}$, and $2.61 \mathrm{~s}$. For the non-optimal layout spatial arrangement of the OWC do not necessarily correspond to a pattern in the average power results over a sweep of wave periods. One explanation for this is the nonlinearities in the system that are not captured in the average of the time series of power produced.

For the optimized layout, in the operating periods of $1.91 \mathrm{~s}, 2.26 \mathrm{~s}$, and $2.61 \mathrm{~s}$, the average power follows a predictable pattern with the center OWC capturing the most and diminishing outward. The numbers inside the lower row of plots represent the average power for the array compared to the non-optimal layouts. In the three operational periods of interest, the data shows a modest increase in power from the non-optimal to optimal layouts.

\section{Irregular wave input}

A similar procedure for irregular waves was performed with the time series shown in Fig. 9 comparing numerical to experimental results. Notice that the phase matches quite well for OWC A,C, and E, and the amplitude of the numerical model matches fairly well. Also notice that OWC B and D do not match in phase or amplitude. This is at least partly explained by the fact that WEC-Sim has the capability for input of only one time series per simulation, whereas an array with practically several input wave conditions is the goal. When there are multiple OWC with different $x$ locations,

\section{Regular Wave - Non-opt Damping - $\mathrm{H}=0.136 \mathrm{~m}$}

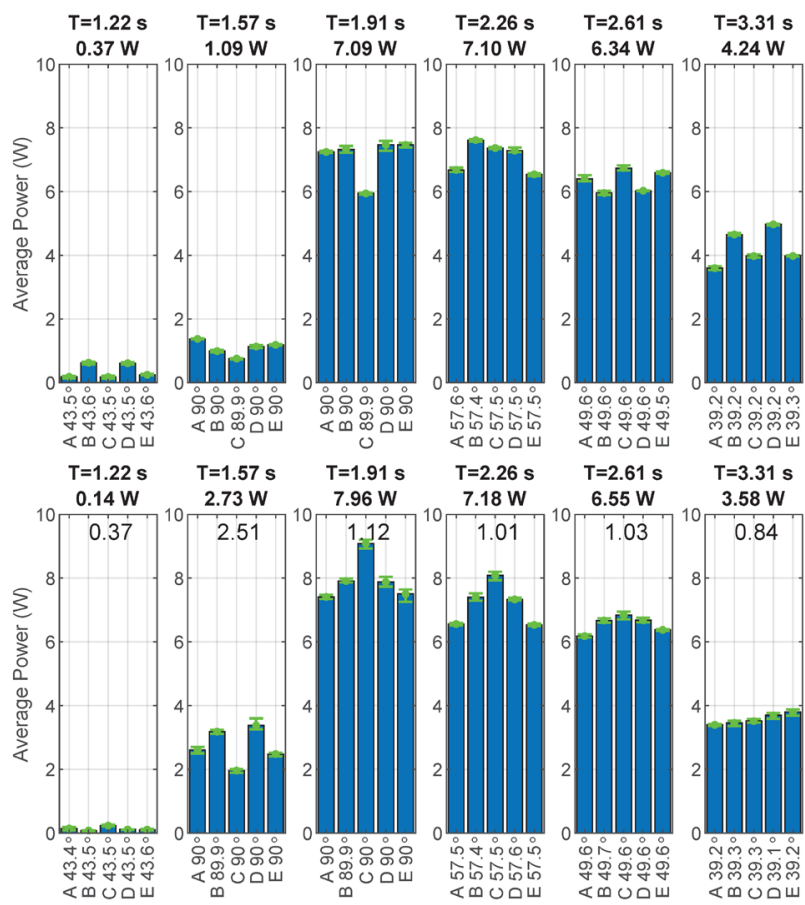

Fig. 8. Subplots of average power for each OWC showing a different period wave input. Top row is non-optimized layout and bottom row is optimized layout. Bar graph represents average of three bridge positions average power. Error bars show minimum and maximum average power of the three bridge positions. Error bars show repeatability in the measurements. The average of the five average powers for the array is shown in the title. The number in the plot in the lower subplots represents the ratio from nonoptimized to optimized layouts, often called the q-factor.

WAMIT/WEC-Sim does not appropriately account for the wave propagation through the tank.

Focusing on average power values for the 600 waves generated for each case, Fig. 10 shows the non-optimal layout in the top row and the optimal layout in the bottom row. The bar plots show the average of three runs corresponding to the three bridge positions. The error bars show the max and min values of the average power resulting from the three bridge positions. Notice the repeatability is quite good for all cases. In the bar plot,

TABLE I

WAVE CONDITIONS TESTED

\begin{tabular}{|c|c|c|c|}
\hline Regular & $H(m)$ & $T(s)$ & \\
\hline 1 & 0.136 & 1.22 & \\
\hline 2 & 0.136 & 1.57 & \\
\hline 3 & 0.136 & 1.91 & \\
\hline 4 & 0.136 & 2.26 & \\
\hline 5 & 0.136 & 2.61 & \\
\hline 6 & 0.136 & 3.31 & \\
\hline Irregular & $H_{m 0}(m)$ & $T_{p}(s)$ & Spread Angle \\
\hline 1 & 0.136 & 1.91 & \\
\hline 2 & 0.136 & 2.26 & \\
\hline 3 & 0.136 & 2.61 & \\
\hline 4 & 0.242 & 3.31 & \\
\hline 5 & 0.136 & 1.91 & $30^{\circ}$ \\
\hline
\end{tabular}

for three bridge positions to capture wave field surrounding OWC. 
Layout Non-optimized and Damping Non-optimized
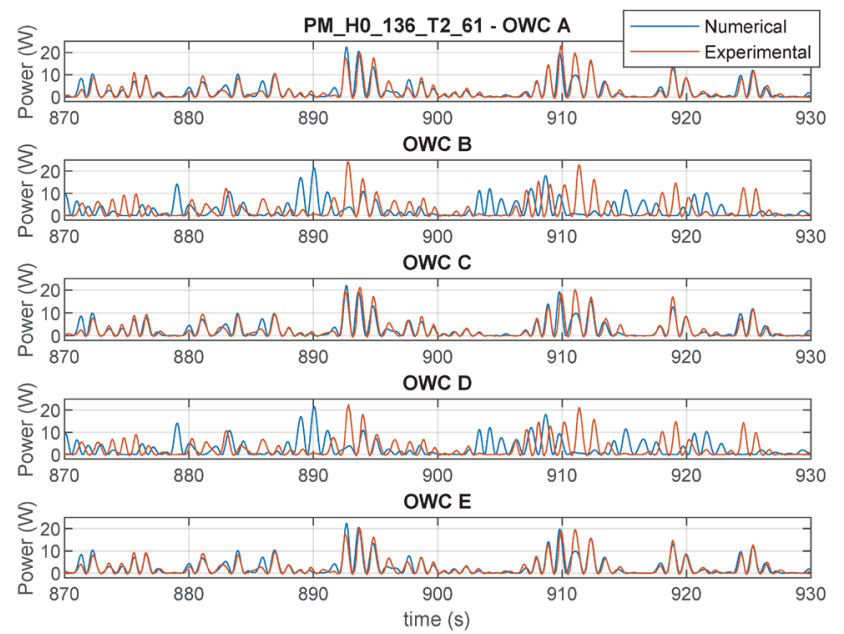

Fig. 10. Comparison of Power time series between experimental and numerical (WEC-Sim) results for a irregular wave with $H_{m 0}=$ $0.136 m, T_{p}=2.61 \mathrm{~s}$. WEC-Sim gives a reasonable estimate for OWC $\mathrm{A}, \mathrm{C}$, and E, however phase and amplitude are off on B and D.

each entry in the $\mathrm{x}$ axis is a different OWC, labelled A-E, followed by the valve angle that the OWC PTO was set to for the duration of the test.

The text in the lower plots shows the ratio of optimallayout to non-optimal layout average power. This shows a slight increase in average power for the most interesting periods of interest, namely 1.91, 2.26, and $2.61 \mathrm{~s}$. Also notice the shape change in the average powers between OWC. Generally, the pattern is symmetric, and for the periods of most interest, the average power seems to benefit slightly from the layout.

\section{CONCLUSION}

This paper outlines the numerical and physical model testing of an array of OWC. Methods of numerical modeling in WAMIT/WEC-Sim are detailed. Physical model testing of two physical layouts of five OWC at the O.H. Hinsdale Wave Research Laboratory is described.

Results are presented for both regular and irregular waves. Numerical and experimental time series are compared showing that WAMIT/WEC-Sim does a fair job of predicting power of the OWC under most conditions. Phase issues arise when there is a physical offset in the direction of wave propagation. Experimental results are shown from the wave tank testing, including regular and irregular average wave power results.

The tests proved to be very repeatable and there was a slight increase in average power for the optimal layout. Results show a max increase of $12 \%$ in average power for regular waves, and $7 \%$ for irregular waves between the non-optimized and optimized layouts. Although the results are clearly different between non-optimal and optimal layouts, interaction effects did not significantly impact absorbed power results. Smaller separation distances between OWC may provide more interaction but would most likely not be practical in a production environment. Future work will include wave by wave control and investigating non-linearities in the system. Additionally, the power results presented in this paper
Irregular Waves Damping Non-optimal

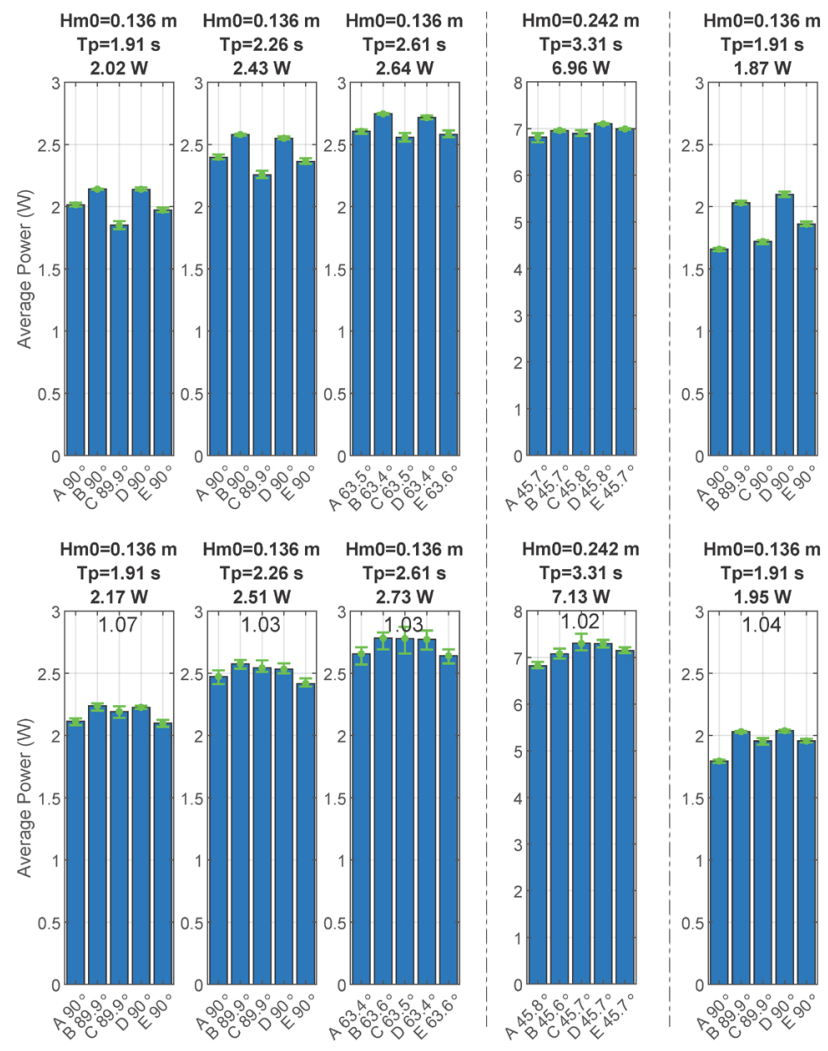

Fig. 9. Subplots of irregular wave average power for each OWC showing a different period wave input. Top row is non-optimized layout and bottom row is optimized layout. The last column shows a short-crested case with a spreading angle of 30 degrees. Bar graph represents average of three bridge positions average power. Error bars show minimum and maximum average power of the three bridge positions. Error bars indicate the repeatability in the measurements. The average of the five average powers for the array is shown in each title. The text number in the plot in the lower subplots represents the ratio of optimized over non-optimized layouts, often called the q-factor.

will be compared to linear frequency domain technique results such as outlined in [18].

\section{REFERENCES}

[1] M. Folley et al., "A Review of Numerical Modelling of Wave Energy Converter Arrays," presented at the ASME 2012 31st International Conference on Ocean, Offshore and Arctic Engineering, 2012, pp. 535-545.

[2] J.-R. Nader, S.-P. Zhu, P. Cooper, and B. Stappenbelt, "A finiteelement study of the efficiency of arrays of oscillating water column wave energy converters," Ocean Eng., vol. 43, pp. 72-81, Apr. 2012

[3] A. Babarit, "On the park effect in arrays of oscillating wave energy converters," Renew. Energy, vol. 58, pp. 68-78, Oct. 2013.

[4] B. F. M. Child and V. Venugopal, "Optimal configurations of wave energy device arrays," Ocean Eng., vol. 37, no. 16, pp. 1402-1417, Nov. 2010.

[5] B. Borgarino, A. Babarit, and P. Ferrant, "Impact of wave interactions effects on energy absorption in large arrays of wave energy converters," Ocean Eng., vol. 41, pp. 79-88, Feb. 2012.

[6] P. Balitsky and V. Stratigaki, "Assessing the Impact on Power Production of WEC array separation distance in a wave farm using one-way coupling of a BEM solver and a wave propagation model," p. 10.

[7] T. K. A. Brekken, H. T. Ozkan-Haller, and A. Simmons, "A Methodology for Large-Scale Ocean Wave Power Time-Series Generation," IEEE J. Ocean. Eng., vol. 37, no. 2, pp. 294-300, Apr. 2012.

[8] M. Göteman, J. Engström, M. Eriksson, and J. Isberg, “Optimizing wave energy parks with over 1000 interacting point-absorbers using 
an approximate analytical method," Int. J. Mar. Energy, vol. 10, pp. 113-126, Jun. 2015.

[9] F. X. Correia da Fonseca, R. P. F. Gomes, J. C. C. Henriques, L. M. C. Gato, and A. F. O. Falcão, "Model testing of an oscillating water column spar-buoy wave energy converter isolated and in array: Motions and mooring forces," Energy, vol. 112, pp. 1207-1218, Oct. 2016.

[10] J.-R. Nader, A. Fleming, G. Macfarlane, I. Penesis, and R. Manasseh, "Novel experimental modelling of the hydrodynamic interactions of arrays of wave energy converters," Int. J. Mar. Energy, vol. 20, pp. 109-124, Dec. 2017.

[11] B. Bosma, T. Brekken, P. Lomonaco, A. McKee, B. Paasch, and B. Batten, "Physical Model Testing and System Identification of a Cylindrical OWC Device," in 12th European Wave and Tidal Energy Conference [accepted], 2017.

[12] C. Sharp, B. DuPont, B. Bosma, P. Lomonaco, and B. Batten, "Array Optimization of Fixed Oscillating Water Columns for Active Device Control," in Proceedings of the Twelfth European Wave and Tidal Energy Conference, University College Cork, Ireland, 2017, pp. 1016 hyphen 1-1016 hyphen 10.

[13] "WEC-Sim (Wave Energy Converter SIMulator) - WEC-Sim documentation." [Online]. Available: https://wec-sim.github.io/WECSim/. [Accessed: 27-Mar-2017].

[14] "Rhino 6 for Windows." [Online]. Available: https://www.rhino3d.com/. [Accessed: 13-Nov-2018].

[15] "Advanced Features - WEC-Sim documentation." [Online]. Available: https://wec-sim.github.io/WECSim/advanced_features.html. [Accessed: 19-Nov-2018].

[16] W. E. Cummins, "The impulse response function and ship motions," DTIC Document, 1962

[17] J. Falnes, Ocean waves and oscillating systems : linear interactions including wave-energy extraction. Cambridge: Cambridge University Press, 2005.

[18] J. C. McNatt, V. Venugopal, and D. Forehand, "A novel method for deriving the diffraction transfer matrix and its application to multibody interactions in water waves," Ocean Eng., vol. 94, pp. 173-185, Jan. 2015. 